\title{
Some Results About Best and 2-Best Approximation on 2-Structures
}

\author{
Mehmet AÇIKGÖZ
}

\begin{abstract}
The problem of best approximation has been studied by many mathematicians. Most of these works have dealt with the existence, uniqueness and characterization of best approximations in spaces of continuous functions with values in Banach spaces. But little or no work on approximation has been done on 2-structures such 2-normed spaces, generalized 2-normed spaces and 2-Banach spaces. It is the aim of this paper to investigate the above two concepts in the sense of latter spaces. It is also to investigate the uniqueness and to give attention of this subject from this view.
\end{abstract}

\section{Introduction, Definitions and Notations}

The concept of linear 2-normed spaces has been investigated by S. Gähler ([1]) and has been developed extensively in different subjects by others (for example, [5] and ([7]-[15])). Then, Lewandowska generalized the notion by providing the notion of generalized 2-normed spaces ([7]-[11]). Also, Rezapour reviewed proximinal subspaces of 2-normed spaces ([16]) and provided the notion of 2-proximinality in 2009 ([17]). The aim of this paper is providing some results in this subjects.

Now, let us give the definition of a 2-normed space which is introduced by S. Gähler in [1].

Definition 1. Let $X$ be a linear space over $K$, where $K$ is the real or complex numbers field, $\operatorname{dim} X>1$, and let

$$
\|., .\|: X^{2} \rightarrow R^{+} \cup\{0\}
$$

be a mapping with the following properties:

(N1) $\|x, y\|=0$ if and only if $x$ and $y$ are linearly dependent vectors;

(N2) $\|x, y\|=\|y, x\|$ for all $x, y \in X$;

(N3) $\|\alpha x, y\|=|\alpha|\|x, y\|, \alpha \in K$ and all $x, y \in X$;

(N4) $\|x+y, z\| \leq\|x, z\|+\|y, z\|$ for all $x, y, z \in X$.

2000 Mathematics Subject Classification. Primary: 46A15; Secondary: 41A65.

Key words and phrases. 2-normed space, Best approximation, 2-best approximation, 2-Banach space. 
Then the mapping $\|.,$.$\| is called a 2$-norm on $X$ and the pair $(X,\|.,\|$. is called a linear 2-normed space. In each 2-normed space $(X,\|.,\|$.$) , we$ have $\|x, y\| \geq 0$ and $\|x, y+\alpha x\|=\|x, y\|$ for all $x, y \in X$ and $\alpha \in R$. Also, if $x, y$ and $z$ are linearly dependent (this occurs for $\operatorname{dim} X=2$ ) then $\|x, y+z\|=\|x, y\|+\|x, z\|$ or $\|x, y-z\|=\|x, y\|+\|x, z\|$.

Every 2-normed space is a locally convex topological vector space. In fact for a fixed $b \in X, p_{b}(x)=\|x, b\|, x \in X$, is a seminorm and the family $P=\left\{p_{b}: b \in X\right\}$ of seminorms generates a locally convex toplogy on $X$. This space will be denoted by $\left(X, p_{b}\right)$.

In [4], it is given some examples and definitions on 2-normed spaces as follows:

Example 1. Let $X=\mathbb{R}^{3}$ and consider the following 2-norm on $X$,

$$
\|x, y\|=|x \times y|=\left|\operatorname{det}\left(\begin{array}{ccc}
i & j & k \\
x_{1} & x_{2} & x_{3} \\
y_{1} & y_{2} & y_{3}
\end{array}\right)\right|
$$

where $x=\left(x_{1}, x_{2}, x_{3}\right)$ and $y=\left(y_{1}, y_{2}, y_{3}\right)$. Then $(X,\|.,\|$.$) is a 2-normed$ space.

Example 2. Let $P_{n}$ denotes the set of real polynomials of degree less than or equal to $n$, on the interval $[0,1]$. By considering usual addition and scalar multiplication, $P_{n}$ is a linear vector space over the reals. Let $\left\{x_{1}, x_{2}, \ldots, x_{2 n}\right\}$ be distinct fixed points in $[0,1]$ and define the 2-norm on $P_{n}$ as

$$
\|f, g\|=\sum_{k=0}^{2 n}\left|f\left(x_{k}\right) \cdot g^{\prime}\left(x_{k}\right)-f^{\prime}\left(x_{k}\right) \cdot g\left(x_{k}\right)\right| .
$$

Then $\left(P_{n},\|.,\|.\right)$ is a linear 2-normed space.

Example 3. Let $X=\mathbb{Q}^{3}$, the field be the rational numbers and consider the norm given in example 1 . In this case $(X,\|.,\|$.$) is a 2$-normed space.

Definition 2. A sequence $\left\{x_{n}\right\}_{n \geq 1}$ in a linear 2-normed space $X$ is called Cauchy sequence if there exist independent elements $y, z \in X$ such that

$$
\lim _{n, m \rightarrow \infty}\left\|x_{n}-x_{m}, y\right\|=0 \text { and } \lim _{n, m \rightarrow \infty}\left\|x_{n}-x_{m}, z\right\|=0 .
$$

Definition 3. A sequence $\left\{x_{n}\right\}_{n \geq 1}$ in a linear 2-normed space $X$ is called convergent if there exists an element $x \in X$ such that $\lim _{n \rightarrow \infty}\left\|x_{n}-x, y\right\|=0$ for all $y \in X$.

Definition 4. Let $(X,\|.,\|$.$) be a 2$-normed space and $W_{1}, W_{2}$ two subspaces of $X$. A map $f: W_{1} \times W_{2} \longrightarrow K$ is called a bilinear 2-functional on $W_{1} \times W_{2}$ whenever for all $x_{1}, x_{2} \in W_{1}, y_{1}, y_{2} \in W_{2}$ and all $\lambda_{1}, \lambda_{2} \in K$;

(i) $f\left(x_{1}+x_{2}, y_{1}+y_{2}\right)=f\left(x_{1}, y_{1}\right)+f\left(x_{1}, y_{2}\right)+f\left(x_{2}, y_{1}\right)+f\left(x_{2}, y_{2}\right)$,

(ii) $f\left(\lambda_{1} x_{1}, \lambda_{2} y_{1}\right)=\lambda_{1} \lambda_{2} f\left(x_{1}, y_{1}\right)$. 
Definition 5. A bilinear 2-functional $f: W_{1} \times W_{2} \longrightarrow K$ is called bounded if there exists a non-negative real number $M$ ( called a Lipschitz constant for $f$ ) such that

$$
|f(x, y)| \leq M\|x, y\|
$$

for all $x \in W_{1}$ and all $y \in W_{2}$. Also, the norm of a bilinear 2-functional $f$ is defined by

$$
\|f\|=\inf \{M \geq 0: M \text { is a Lipschitz constant for } f\} .
$$

For a 2-normed space $(X,\|.,\|$.$) , a subspace W$ of $X$ and $b \in X$, we denote by $W_{b}^{\sharp}$; the Banach space of all bounded bilinear 2-functionals on $W \times\langle b\rangle$.

Definition 6 ([10]). Let $X$ and $Y$ be linear spaces, $D$ be non-empty subset of $X \times Y$ such that for every $x \in X, y \in Y$ the sets

$$
D_{x}=\{y \in Y:(x, y) \in D\}, \quad D^{y}=\{x \in X:(x, y) \in D\}
$$

are linear subspaces of $Y$ and $X$, respectively. A function $\|.,\|:. D \rightarrow[0, \infty)$ is called a generalized 2-norm on $D$ if it satisfies the following conditions:

(G2N1) $\|x, \alpha y\|=|\alpha| .\|x, y\|=\|\alpha x, y\|$ for all $(x, y) \in D$ and every scalar $\alpha$; (G2N2) $\|x, y+z\|=\|x, y\|+\|x, z\| \quad$ for all $(x, y),(x, z) \in D$;

(G2N3) $\|x+y, z\|=\|x, z\|+\|y, z\| \quad$ for all $(x, z),(y, z) \in D$.

Then $(D,\|.,\|$.$) is called a 2-normed set. In particular, if it is taken as$ $D=X \times Y$, then $(X \times Y,\|.,\|$.$) is called a generalized 2-normed space.$ In addition, if $X=Y$, then the generalized 2-normed space is denoted by $(X,\|.,\|$.$) or (Y,\|.,\|$.$) .$

There are some another definitions about Cauchy sequences and convergent in 2-normed spaces.

Definition $7([9]) . \quad(i)$ A sequence $\left\{x_{n}\right\}_{n \geq 1}$ in a 2-normed space $(X,\|.,\|$.$) is called a convergent if there exists x \in X$ such that $\left\{\left\|x_{n}-x, y\right\|\right\}_{n \geq 1}$ tends to zero for all $y \in X$. In this case, we write $\lim _{n \rightarrow \infty} x_{n}=x$ and we call $x$ the limit of $\left\{x_{n}\right\}_{n \geq 1}$. The uniqueness of the limit of a convergent can be shown as follows. To show this, suppose $\left\{x_{n}\right\}_{n \geq 1}$ is convergent to two distinct limits $x$ and $y$ in $X$. For this choose $z \in X$ such that $\|x-y, z\| \neq 0$ and taking $n_{0} \in N$ sufficiently large such that $\left\|x_{n_{0}}-x, z\right\|<\frac{1}{2}\|x-y, z\|$ and $\left\|x_{n_{0}}-y, z\right\|<\frac{1}{2}\|x-y, z\|$ simultaneously. Then using the triangle inequality, we get

$$
\begin{aligned}
\|x-y, z\| & \leq\left\|x-x_{n_{0}}, z\right\|+\left\|x_{n_{0}}-y, z\right\| \\
& <\frac{1}{2}\|x-y, z\|+\frac{1}{2}\|x-y, z\| \\
& =\|x-y, z\|
\end{aligned}
$$

which is contradiction. Hence, whenever limit exists, it must be unique. 
(ii) A sequence $\left\{x_{n}\right\}_{n \geq 1}$ in a 2-normed space $(X,\|.,\|$.$) is called a Cauchy$ sequence if there exist two linearly independent elements $y$ and $z$ in $X$ such that $\left\{\left\|x_{n}, y\right\|\right\}_{n \geq 1}$ and $\left\{\left\|x_{n}, z\right\|\right\}_{n \geq 1}$ are real Cauchy sequences.

Definition 8. A 2-normed space $(X,\|.,\|$.$) is called 2-Banach space if every$ Cauchy sequence is convergent.

The Examples 1 and 2 are 2-Banach spaces while the Example 3 does not. (For details, see [4]).

Lemma 1 ([4]). (i) Every 2-normed space of dimension 2 is a 2-Banach space, when the underlying field is complete.

(ii) If $\left\{x_{n}\right\}$ is a sequence in 2-normed space $(X,\|.,\|$.$) and$ $\lim _{n \rightarrow \infty}\left(\left\|x_{n}-x, y\right\|\right)=0$, then $\lim _{n \rightarrow \infty}\left\|x_{n}, y\right\|=\|x, y\|$.

A sequence $\left(x_{n}\right)$ in $X$ is said to be convergent to $x$ in $X$ if

$$
\lim _{n \rightarrow \infty}\left\|x_{n}-x, y\right\|=0
$$

for every $y \in X$.

We conclude this section by a known lemma needed in the proof of a main result.

Proposition 1 ([12];Theorem 3.6). Let $(X,\|.,\|$.$) be a linear 2-normed$ space, $W$ be a subspace of $X, b \in X$ and let $\langle b\rangle$ be the subspace of $X$ generated by $b$. If $x_{0} \in X$ is such that

$$
\delta=\inf \left\{\left\|x_{0}-w, b\right\|: w \in W\right\}>0,
$$

then there exists a bounded bilinear functional $F: X \times\langle b\rangle \rightarrow K$ such that $\left.F\right|_{W \times\langle b\rangle}=0, F\left(x_{0}, b\right)=1$ and $\|F\|=\frac{1}{\delta}$.

\section{Some Types of Proximinality in 2-NORmed SPACES}

The main part of this paper is given in this section. We know that, approximation is an old notion. It has many applications in many areas, especially in engineering. Here, we will denote by $\langle b\rangle$ be the subspace of $X$ generated by $b$, by $P_{W}^{b}(x)$, the set of all $b$-best approximations of $x$ in $W$ and by $X_{b}^{\sharp}$, the Banach space of all bounded bilinear 2-functionals on $W \times\langle b\rangle$. Also, we will provide some results about approximation in generalized 2-normed spaces [3]. The following definitions are important in establishing our results, theorems, corollaries and examples.

Definition 9. Let $(X,\|.,\|$.$) be a 2-normed space, W$ be a subspace of $X$, $0 \neq b \in X$ and let $\langle b\rangle$ be the subspace of $X$ generated by $b$.

(i) $w_{0} \in W$ is called b-best approximation of $x \in X$ in $W$, if

$$
\left\|x-w_{0}, b\right\|=\inf \{\|x-w, b\|: \quad w \in W\} .
$$

The set of all $b$-best approximations of $x$ in $W$ is denoted by $P_{W}^{b}(x)$. 
(ii) $W$ is called $b$-proximinal if for every $x \in X \backslash(\bar{W} \backslash W)$, there exists $w_{0} \in W$ such that

$$
\left\|x-w_{0}, b\right\|=\inf \{\|x-w, b\|: \quad w \in W\},
$$

where $\bar{W}$ denotes the closure of $W$ in the seminormed space $\left(X, p_{b}\right)$.

(iii) $W$ is called 1-type proximinal if $W$ is b-proximinal for all $0 \neq b \in X$, that is for every $0 \neq b \in X$ and every $x \in X \backslash(\bar{W}+\langle b\rangle)$, where $\bar{W}$ denotes the closure of $W$ in the seminormed space $\left(X, p_{b}\right)$, there exists $w_{0} \in W$ such that

$$
\left\|x-w_{0}, b\right\|=\inf \{\|x-w, b\|: \quad w \in W\} .
$$

Definition 10. (i) Let $(X \times Y,\|.,\|$.$) be a generalized 2-normed space,$ $W_{1}$ be a subspace of $X$ and let $W_{2}$ be a subspace of $Y$. Then, $W_{1} \times W_{2}$ is called 2-proximinal if for every $(x, y) \in X \times Y$ there exists $\left(w_{0}, g_{o}\right) \in W_{1} \times W_{2}$ such that

$$
\left\|x-w_{0}, y-g_{0}\right\|=\inf \left\{\|x-w, y-g\|:(w, g) \in W_{1} \times W_{2}\right\} .
$$

In this case, $\left(w_{0}, g_{0}\right)$ is called 2-best approximation of $(x, y)$ in $W_{1} \times W_{2}$ and the set of all 2-best approximations of $(x, y)$ in $W_{1} \times W_{2}$ is denoted by $P_{W_{1} \times W_{2}}^{2}(x, y)$.

(ii) Let $(X \times Y,\|.,\|$.$) be a generalized 2-normed space and f$ be a realvalued map on $X \times Y$. Then, $f$ is called a 2 -subadditive if

$$
\begin{aligned}
f\left(x_{1}+x_{2}, y\right) & \leq f\left(x_{1}, y\right)+f\left(x_{2}, y\right), \\
f\left(x, y_{1}+y_{2}\right) & \leq f\left(x, y_{1}\right)+f\left(x, y_{2}\right)
\end{aligned}
$$

for all $x, x_{1}, x_{2} \in X$ and all $y, y_{1}, y_{2} \in Y$.

Also, $f$ is called bounded if there exists a positive real number $M$ such that $|f(x, y)| \leq M\|x, y\|$, for all $(x, y) \in X \times Y$. Then, the norm of $f$ is defined by

$$
\|f\|=\inf \{M>0:|f(x, y)| \leq M\|x, y\|,
$$

for all $(x, y) \in X \times Y\}$.

Note that, $|f(x, y)| \leq\|f\|\|x, y\|$, for all $(x, y) \in X \times Y$.

\subsection{Some Results.}

(1) If $b \in W$ and $\operatorname{dim} W=1$, then $P_{W}^{b}(x)=W$ for all $x \in X$.

(2) If $x \in W$, then $P_{W}^{b}(x)=x+\langle b\rangle$.

(3) If $x$ is not in $W+\langle b\rangle$, then $P_{W}^{b}(x)=\emptyset$ for all $x \in \bar{W} \backslash W$, where $\bar{W}$ denotes the closure of $W$ in the seminormed space $\left(X, p_{b}\right)$.

(4) If $x \in W+\langle b\rangle$ and $x=w_{1}+\lambda_{1} b$, then $P_{W}^{b}(x)=\left\{w_{1}\right\}$.

(5) If $x \in\langle b\rangle$, then $P_{W}^{b}(x)=W \cap\langle b\rangle$.

(6) $P_{W}^{b}(x)$ is closed and convex in $\left(X, p_{b}\right)$, for all $x \in X$. 
Finally note that, $W$ is b-proximinal if and only if $P_{W}^{b}(x) \neq \emptyset$ for all $x \in X \backslash(\bar{W}+\langle b\rangle)$.

Theorem 1. Let $(X,\|.,\|$.$) be a 2-normed space, W$ be a subspace of $X$, $0 \neq b \in X, w_{0} \in W$ and let $\langle b\rangle$ be the subspace of $X$ generated by $b$. Suppose that $x_{0} \in X$ is such that

$$
\delta=\inf \left\{\left\|x_{0}-w, b\right\|: w \in W\right\}>0 .
$$

Then, $w_{0} \in P_{W}^{b}\left(x_{0}\right)$ if and only if there exists $f \in X_{b}^{\sharp}$ such that $\left.f\right|_{W \times\langle b\rangle}=0$, $f\left(x_{0}-w_{0}, b\right)=\left\|x_{0}-w_{0}, b\right\|$ and $\|f\|=1$.

Proof. First suppose that there exists $f \in X_{b}^{\sharp}$ such that $\left.f\right|_{W \times\langle b\rangle}=0$, $f\left(x_{0}-w_{0}, b\right)=\left\|x_{0}-w_{0}, b\right\|$ and $\|f\|=1$. Then,

$$
\begin{gathered}
\left\|x_{0}-w_{0}, b\right\|=f\left(x_{0}-w_{0}, b\right)=f\left(x_{0}, b\right)=f\left(x_{0}-w, b\right) \\
\leq\|f\|\left\|x_{0}-w, b\right\|=\left\|x_{0}-w, b\right\|,
\end{gathered}
$$

for all $w \in W$. Hence, $w_{0} \in P_{W}^{b}\left(x_{0}\right)$. Conversely, let $w_{0} \in P_{W}^{b}\left(x_{0}\right)$. Then, $\delta=\left\|x_{0}-w_{0}, b\right\|=\inf \left\{\left\|x_{0}-w, b\right\|: w \in W\right\}>0$. By Proposition 2, there exists $g \in X_{b}^{\sharp}$ such that $\left.g\right|_{W \times\langle b\rangle}=0, g\left(x_{0}, b\right)=1$ and $\|f\|=\frac{1}{\delta}$. Now for $f=\delta g$ we have, $\left.f\right|_{W \times\langle b\rangle}=0, f\left(x_{0}-w_{0}, b\right)=\left\|x_{0}-w_{0}, b\right\|$ and $\|f\|=1$.

Corollary 1. Let $(X,\|.,\|$.$) be a 2-normed space, W$ be a subspace of $X$, $0 \neq b \in X$ and let $\langle b\rangle$ be the subspace of $X$ generated by $b$. Then, $W$ is $b$-proximinal subspace of $X$ if and only if for every $x \in X \backslash(\bar{W}+\langle b\rangle)$, where $\bar{W}$ denotes the closure of $W$ in the seminormed space $\left(X, p_{b}\right)$, there exist $w_{0} \in W$ and $f \in X_{b}^{\sharp}$ such that $\left.f\right|_{W \times\langle b\rangle}=0, f\left(x-w_{0}, b\right)=\left\|x-w_{0}, b\right\|$ and $\|f\|=1$.

Lemma 2. Let $(X,\|.,\|$.$) be a 2-normed space, W$ be a subspace of $X$, $0 \neq b \in X, x \in X \backslash(\bar{W}+[b])$, where $\bar{W}$ denotes the closure of $W$ in the seminormed space $\left(X, p_{b}\right)$, and let $\langle b\rangle$ be the subspace of $X$ generated by $b$. Then, $M \subseteq P_{W}^{b}(x)$ if and only if there exists $f \in X_{b}^{\sharp}$ such that $\left.f\right|_{W \times[b]}=0$, $\|f\|=1$ and $f\left(x_{0}-m, b\right)=\left\|x_{0}-m, b\right\|$ for all $m \in M$.

Proof. Let $M \subseteq P_{W}^{b}(x)$ and fix $m_{1} \in M$. By Theorem 1, there exists $f \in X_{b}^{\sharp}$ such that $\left.f\right|_{W \times\langle b\rangle}=0, f\left(x_{0}-m_{1}, b\right)=\left\|x_{0}-m_{1}, b\right\|$ and $\|f\|=1$. But, $f\left(x_{0}-m, b\right)=\left\|x_{0}-m_{1}, b\right\|=\left\|x_{0}-m, b\right\|$, for all $m \in M$.

Example 4. Let $X=\mathbb{R}^{2}$, the plane, $W=\{(x, y) \in X: x=y\}$ and $\left\|\left(x_{1}, x_{2}\right),\left(y_{1}, y_{2}\right)\right\|=\left|x_{1} y_{2}-x_{2} y_{1}\right|$ for all $\left(x_{1}, x_{2}\right),\left(y_{1}, y_{2}\right) \in X$. Let $b=$ $\left(b_{1}, b_{2}\right) \in X \backslash\{(0,0)\}$. Then, $\|.,$.$\| is a 2$-norm on $X, W$ is b-proximinal subspace of $X, P_{W}^{b}(x)=W$ if $b \in W$ and $P_{W}^{b}(x)=\left\{\left(\frac{x_{2} b_{1}-x_{1} b_{2}}{b_{2}-b_{1}}, \frac{x_{2} b_{1}-x_{1} b_{2}}{b_{2}-b_{1}}\right)\right\}$ if $b$ is not in $W$. 
Example 5. Let $X=\mathbb{R}^{3}, W=\{(0, x, 0): x \in \mathbb{R}\}$ and

$$
\left\|\left(x_{1}, x_{2}, x_{3}\right),\left(y_{1}, y_{2}, y_{3}\right)\right\|=\left|x_{1} y_{2}-x_{2} y_{1}\right|+\left|x_{2} y_{3}-x_{3} y_{2}\right|+\left|x_{1} y_{3}-x_{3} y_{1}\right|
$$

for all $\left(x_{1}, x_{2}, x_{3}\right),\left(y_{1}, y_{2}, y_{3}\right) \in X$. Let $b=\left(b_{1}, b_{2}, b_{3}\right) \in X \backslash W$. Then, $\|.,$.$\| is a 2-norm on X$ and $W$ is b-proximinal subspace of $X$. In fact, $P_{W}^{b}(x)=\left\{\left(0, \frac{x_{2} b_{3}-x_{3} b_{2}}{b_{3}}, 0\right)\right\}$ if $b_{1}=0$ and $b_{3} \neq 0, P_{W}^{b}(x)=\left\{\left(0, \frac{x_{2} b_{1}-x_{1} b_{2}}{b_{1}}, 0\right)\right\}$ if $b_{1} \neq 0$ and $b_{3}=0, P_{W}^{b}(x)=\left\{\left(0, \frac{x_{2} b_{1}-x_{1} b_{2}}{b_{1}}, 0\right)\right\}$ if $b_{1} \neq 0, b_{3} \neq 0$ and $\left|-b_{1} b_{3} x_{3}+b_{3}^{2} x_{1}\right| \leq\left|b_{1} b_{3} x_{1}-b_{1}^{2} x_{3}\right|$, and finally $P_{W}^{b}(x)=\left\{\left(0, \frac{x_{2} b_{3}-x_{3} b_{2}}{b_{3}}, 0\right)\right\}$ if $b_{1} \neq 0, b_{3} \neq 0$ and $\left|-b_{1} b_{3} x_{3}+b_{3}^{2} x_{1}\right| \geq\left|b_{1} b_{3} x_{1}-b_{1}^{2} x_{3}\right|$. Since $\operatorname{dim} W=1$, $P_{W}^{b}(x)=W$ if $0 \neq b \in W$.

Theorem 2. Let $(X,\|.,\|$.$) be a 2-normed space and let W$ be a subspace of $X$. Then, $W$ is 1-type proximinal subspace of $X$ if and only if for every $0 \neq b \in X$ and every $x \in X \backslash(\bar{W}+\langle b\rangle)$, where $\bar{W}$ denotes the closure of $W$ in the seminormed space $\left(X, p_{b}\right)$, there exist $w_{0} \in W$ and $f_{b} \in X_{b}^{\sharp}$ such that $\left.f_{b}\right|_{W \times\langle b\rangle}=0, f_{b}\left(x-w_{0}, b\right)=\left\|x-w_{0}, b\right\|$ and $\left\|f_{b}\right\|=1$.

Proof. Note that, $\delta=\inf \left\{\left\|x_{0}-w, b\right\|: w \in W\right\}>0$, whenever $x \in$ $X \backslash(\bar{W}+\langle b\rangle)$. Hence, it is an immediate consequence of Theorem 1 .

Definition 11. Let $(X,\|.,\|$.$) be a 2-normed space, E$ be a subset of $X$ and $0 \neq b \in X$. An element $x \in X$ is said to be b-orthogonal to an element $y \in X$, and we write $x \perp_{b} y$, if $\|x+\lambda y, b\| \geq\|x, b\|$ for every scalar $\lambda$. Also, an element $x \in X$ is said to be orthogonal to $E$, and we write $x \perp_{b} E$, if $x \perp_{b} y$ for all $y \in E$.

Lemma 3. Let $(X,\|.\|$,$) be a 2-normed space, W$ be a subspace of $X, b \in X$. Then, $w_{0} \in P_{W}^{b}(x)$ if and only if $x-w_{0} \perp_{b} W$.

Proof. Note that, $\left\|x-w_{0}+\lambda w, b\right\| \geq\left\|x-w_{0}, b\right\|$, for all $w \in W$ and every scalar $\lambda$ if and only if $w_{0} \in P_{W}^{b}(x)$.

Corollary 2. Let $(X,\|.,\|$.$) be a 2-normed space and let W$ be a subspace of $X$. Then, $W$ is 1-type proximinal subspace of $X$ if and only if for every $0 \neq b \in X$ and every $x \in X \backslash(\bar{W}+\langle b\rangle)$, where $\bar{W}$ denotes the closure of $W$ in the seminormed space $\left(X, p_{b}\right)$, there exist $w_{0} \in W$ such that $x-w_{0} \perp_{b} W$.

Lemma 4. Let $(X,\|.,\|$.$) be a 2-normed space, W$ be a subspace of $X$, $0 \neq b \in X$ and $\bar{W}$ denotes the closure of $W$ in the seminormed space $\left(X, p_{b}\right)$. Then the following are equivalent:

(1) $W$ is b-proximinal.

(2) $W+\langle b\rangle$ is closed in $\left(X, p_{b}\right)$ and for every $x \in X \backslash(\bar{W}+\langle b\rangle)$, there exists an element $0 \neq y_{0} \in W_{x}=W \bigoplus\langle x\rangle$ such that $y_{0} \perp_{b} W$.

(3) $W+\langle b\rangle$ is closed in $\left(X, p_{b}\right)$ and for every $x \in X \backslash(\bar{W}+\langle b\rangle)$, every $\varphi \in\left(W_{x}\right)_{b}^{\#}$ with the property

$$
W=\left\{y \in W_{x}: \varphi(y)=0\right\}
$$


has at least one maximal element, that is, $z \in W_{x} \backslash\{0\}$ such that $\varphi(z, b)=\|\varphi\|\|z, b\|$.

Proof. (1) $\Rightarrow(2)$ Let $\left\{g_{n}+\lambda_{n} b\right\}_{n \geq 1}$ be a sequence in $W+\langle b\rangle$ and $g_{n}+$ $\lambda_{n} b \longrightarrow x_{0}$ for some $x_{0} \in X$. Choose $g_{0} \in P_{W}^{b}\left(x_{0}\right)$. Then, $\left\|x_{0}-g_{0}, b\right\| \leq$ $\left\|x_{0}-g_{n}, b\right\|=\left\|x_{0}-g_{n}-\lambda_{n} b, b\right\| \longrightarrow 0$. Hence, $x_{0} \in W+\langle b\rangle$. Now, for every $x \in X \backslash(\bar{W}+\langle b\rangle)$, take $g_{0} \in P_{W}^{b}(x)$. Then, $0 \neq y_{0}=x-g_{0} \in W_{x}$ and $y_{0} \perp_{b} W$.

$(2) \Rightarrow(3)$ For every $x \in X \backslash(\bar{W}+\langle b\rangle)$, there exists an element $0 \neq y_{0} \in W_{x}$ such that $y_{0} \perp_{b} W$. Then, $0 \in P_{W}^{b}\left(y_{0}\right)$. Thus by Theorem 1 , there exists $\psi \in\left(W_{x}\right)_{b}^{\sharp}$ such that $\|\psi\|=1,\left.\psi\right|_{W \times\langle b\rangle}=0, \psi\left(y_{0}, b\right)=\left\|y_{0}, b\right\|$. Let now $\varphi \in\left(W_{x}\right)_{b}^{\sharp} \backslash\{0\}$ be arbitrary with the property $W=\left\{y \in W_{x}: \varphi(y)=0\right\}$. Then, there exists a non-zero scalar $\lambda$ such that $\varphi=\lambda \psi$. Hence,

$$
\begin{aligned}
\varphi\left(\bar{\lambda} y_{0}, b\right) & =(\lambda \psi)\left(\bar{\lambda} y_{0}, b\right)=|\lambda|^{2} \psi\left(y_{0}, b\right) \\
& =|\lambda|^{2}\left\|y_{0}, b\right\|=\|\lambda \psi\|\left\|\bar{\lambda} y_{0}, b\right\|=\|\varphi\|\left\|\bar{\lambda} y_{0}, b\right\| .
\end{aligned}
$$

Therefore, $\bar{\lambda} y_{0}$ is a maximal element of $\varphi$.

$(3) \Rightarrow(1)$ For every $x \in X \backslash(\bar{W}+\langle b\rangle)$, choose $\varphi \in\left(W_{x}\right)_{b}^{\sharp}$ such that $W=\left\{y \in W_{x}: \varphi(y)=0\right\}$ and $0 \neq z \in W_{x}$ such that $\varphi(z, b)=\|\varphi\|\|z, b\|$. Put, $\psi=\frac{\varphi}{\|\varphi\|}$. Then, $\|\psi\|=1,\left.\psi\right|_{W \times\langle b\rangle}=0, \psi(z, b)=\|z, b\|$. By Theorem $1,0 \in P_{W_{x}}^{b}(z)$. Now, put $w_{0}=x-\frac{\varphi(x, b)}{\varphi(z, b)} z$. Note that $w_{0} \in W$, because $\varphi\left(w_{0}, b\right)=0$. Also, $\frac{\varphi(z, b)}{\varphi(x, b)}\left(w-w_{0}\right) \in W$ for all $w \in W$. On the other hand, $\left\|x-w_{0}, b\right\|=\left|\frac{\varphi(x, b)}{\varphi(z, b)}\right|\|z, b\| \leq\left|\frac{\varphi(x, b)}{\varphi(z, b)}\right|\left\|z-\frac{\varphi(z, b)}{\varphi(x, b)}\left(w-w_{0}\right), b\right\|=\|x-w, b\|$. Therefore, $w_{0} \in P_{W}^{b}(x)$.

Theorem 3. Let $(X \times Y,\|.\|$,$) be a generalized 2-normed space, W_{1}$ be a subspace of $X, W_{2}$ be a subspace of $Y,\left(w_{0}, g_{0}\right) \in W_{1} \times W_{2}$ and $(x, y) \in X \times Y$. Then, $\left(w_{0}, g_{0}\right) \in P_{W_{1} \times W_{2}}^{2}(x, y)$ if and only if there exists a 2-subadditive map $f: X \times Y \longrightarrow \mathbb{R}$ such that $\left.f\right|_{W_{1} \times\{y\}}=\left.f\right|_{\{x\} \times W_{2}}=\left.f\right|_{W_{1} \times W_{2}}=0,\|f\| \leq 1$ and $f\left(x-w_{0}, y-g_{0}\right)=\left\|x-w_{0}, y-g_{0}\right\|$.

Proof. First suppose that there exists a 2-subadditive map $f: X \times Y \longrightarrow \mathbb{R}$ such that $\left.f\right|_{W_{1} \times\{y\}}=\left.f\right|_{\{x\} \times W_{2}}=\left.f\right|_{W_{1} \times W_{2}}=0,\|f\| \leq 1$ and $f\left(x_{0}-w_{0}, y-\right.$ $\left.g_{0}\right)=\left\|x_{0}-w_{0}, y-g_{0}\right\|$. Then, $f\left(x-w_{0}, y-g_{0}\right)=f(x-w, y-g)=f(x, y)$ for all $w \in W_{1}$ and all $g \in W_{2}$. Hence,

$$
\begin{aligned}
\left\|x-w_{0}, y-g_{0}\right\| & =f\left(x-w_{0}, y-g_{0}\right)=f(x-w, y-g) \\
& \leq|f(x-w, y-g)| \leq\|f\|\|x-w, y-g\| \\
& \leq\|x-w, y-g\|,
\end{aligned}
$$

for all $w \in W_{1}$ and all $g \in W_{2}$. Therefore, $\left(w_{0}, g_{0}\right) \in P_{W_{1} \times W_{2}}^{2}(x, y)$. 
Now, let $\left(w_{0}, g_{0}\right) \in P_{W_{1} \times W_{2}}^{2}(x, y)$. Define $f: X \times Y \longrightarrow \mathbb{R}$ by $f(x, y)=$ $\inf _{w \in W_{1}, g \in W_{2}}\{\|x-w, y-g\|\}$. It is easy to see that $f$ is a 2-subadditive map such that $\left.f\right|_{W_{1} \times\{y\}}=\left.f\right|_{\{x\} \times W_{2}}=\left.f\right|_{W_{1} \times W_{2}}=0$. Also, $\|f\| \leq 1$ because $|f(x, y)|=f(x, y) \leq\|x, y\|$ for all $(x, y) \in X \times Y$. Finally,

$$
\begin{aligned}
f\left(x-w_{0}, y-g_{0}\right) & =\inf _{w \in W_{1}, g \in W_{2}}\left\{\left\|x-w_{0}-w, y-g_{0}-g\right\|\right\} \\
& =\inf _{t \in W_{1}, s \in W_{2}}\{\|x-t, y-s\|\} \\
& =\left\|x-w_{0}, y-g_{0}\right\| .
\end{aligned}
$$

Corollary 3. Let $(X \times Y,\|.,\|$.$) be a generalized 2-normed space, W_{1}$ be a subspace of $X$ and $W_{2}$ be a subspace of $Y$. Then, $W_{1} \times W_{2}$ is 2-proximinal subspace of $X$ if and only if for every $(x, y) \in X \times Y$ there exists a 2subadditive map $f$ on $X \times Y$ such that $\left.f\right|_{W_{1} \times\{y\}}=\left.f\right|_{\{x\} \times W_{2}}=\left.f\right|_{W_{1} \times W_{2}}=0$, $\|f\| \leq 1$ and $f\left(x-w_{0}, y-g_{0}\right)=\left\|x-w_{0}, y-g_{0}\right\|$.

Lemma 5. Let $(X \times Y,\|.,\|$.$) be a generalized 2-normed space, W_{1}$ be a subspace of $X, W_{2}$ be a subspace of $Y$ and $(x, y) \in X \times Y$. Then, $M \subseteq$ $P_{W_{1} \times W_{2}}^{2}(x, y)$ if and only if there exists a 2-subadditive map $f$ on $X \times Y$ such that

and

$$
\left.f\right|_{W_{1} \times\{y\}}=\left.f\right|_{\{x\} \times W_{2}}=\left.f\right|_{W_{1} \times W_{2}}=0, \quad\|f\| \leq 1
$$

for all $\left(m_{1}, m_{2}\right) \in M$.

$$
f\left(x-m_{1}, y-m_{2}\right)=\left\|x-m_{1}, y-m_{2}\right\|
$$

Proof. Let $M \subseteq P_{W_{1} \times W_{2}}^{2}(x, y)$ and fix $\left(t_{1}, t_{2}\right) \in M$. By Theorem 1 , there exists a 2-subadditive map in each variable $f$ on $X \times Y$ such that

$$
\left.f\right|_{W_{1} \times\{y\}}=\left.f\right|_{\{x\} \times W_{2}}=\left.f\right|_{W_{1} \times W_{2}}=0, \quad\|f\| \leq 1
$$

and

$$
f\left(x-t_{1}, y-t_{2}\right)=\left\|x-t_{1}, y-t_{2}\right\| .
$$

But

$$
f\left(x-t_{1}, y-t_{2}\right)=f\left(x-m_{1}, y-m_{2}\right)
$$

for all $\left(m_{1}, m_{2}\right) \in M$. Therefore,

$$
f\left(x-m_{1}, y-m_{2}\right)=\left\|x-t_{1}, y-t_{2}\right\|=\left\|x-m_{1}, y-m_{2}\right\|
$$

for all $\left(m_{1}, m_{2}\right) \in M$.

Example 6. Let $X=Y=\mathbb{R}^{2}, W_{1}=\left\{\left(x_{1}, x_{2}\right) \in X: x_{1}=x_{2}\right\}$, $W_{2}=\left\{\left(y_{1}, y_{2}\right) \in Y: y_{1}=y_{2}\right\}$ and define $\|.,\|:. X \times Y \longrightarrow \mathbb{R}$ by

$$
\left\|\left(x_{1}, x_{2}\right),\left(y_{1}, y_{2}\right)\right\|=\left|x_{1} y_{2}-x_{2} y_{1}\right|
$$

for all $\left(x_{1}, x_{2}\right) \in X,\left(y_{1}, y_{2}\right) \in Y$. Then, $\|.,$.$\| is a generalized 2-norm on$ $X \times Y$ and $W_{1} \times W_{2}$ is 2-proximinal subspace of $X \times Y$. In fact,

$$
P_{W_{1} \times W_{2}}^{2}(x, y)=W_{1} \times\left\{\left(\frac{x_{1} y_{2}-x_{2} y_{1}}{x_{1}-x_{2}}, \frac{x_{1} y_{2}-x_{2} y_{1}}{x_{1}-x_{2}}\right)\right\}
$$


if $y_{1}=y_{2}$ and $x_{1} \neq x_{2}$;

$$
P_{W_{1} \times W_{2}}^{2}(x, y)=\left\{\left(\frac{x_{1} y_{2}-x_{2} y_{1}}{y_{2}-y_{1}}, \frac{x_{1} y_{2}-x_{2} y_{1}}{y_{2}-y_{1}}\right)\right\} \times W_{2},
$$

if $x_{1}=x_{2}$ and $y_{1} \neq y_{2} ; P_{W_{1} \times W_{2}}^{2}(x, y)=W_{1} \times W_{2}$, if $x_{1}=x_{2}$ and $y_{1}=y_{2}$; and finally

$$
\begin{aligned}
& \left(\frac{x_{1} y_{2}-x_{2} y_{1}}{y_{2}-y_{1}}, \frac{x_{1} y_{2}-x_{2} y_{1}}{y_{2}-y_{1}}, 0,0\right), \\
& \left(0,0, \frac{x_{1} y_{2}-x_{2} y_{1}}{x_{1}-x_{2}}, \frac{x_{1} y_{2}-x_{2} y_{1}}{x_{1}-x_{2}}\right) \in P_{W_{1} \times W_{2}}^{2}(x, y)
\end{aligned}
$$

if $x_{1} \neq x_{2}$ and $y_{1} \neq y_{2}$.

Example 7. Let $W_{1}$ and $W_{2}$ be proximinal subspaces of $\left(X,\|\cdot\|_{1}\right)$ and $\left(Y,\|\cdot\|_{2}\right)$, respectively. Then, $\|x, y\|=\|x\|_{1}\|y\|_{2}$ is a generalized 2-norm on $X \times Y$ and $P_{W_{1}}(x) \times P_{W_{2}}(y) \subseteq P_{W_{1} \times W_{2}}^{2}(x, y)$ for all $(x, y) \in X \times Y$. Also, the equality holds if $x \in X \backslash W_{1}$ and $y \in Y \backslash W_{2}$.

Definition 12. Let $(X \times Y,\|.,\|$.$) be a generalized 2-normed space and let E$ be a subset of $X \times Y$. An element $(x, y) \in X \times Y$ is said to be orthogonal to an element $(t, s) \in X \times Y$, and we write $(x, y) \perp(t, s)$, if $\left\|x+\lambda_{1} t, y+\lambda_{2} s\right\| \geq$ $\|x, y\|$ for all scalars $\lambda_{1}$ and $\lambda_{2}$. Also, an element $(x, y) \in X \times Y$ is said to be orthogonal to $E$, and we write $(x, y) \perp E$, if $(x, y) \perp(t, s)$ for all $(t, s) \in E$.

Lemma 6. Let $(X \times Y,\|.,\|$.$) be a generalized 2-normed space, W_{1}$ be a subspace of $X, W_{2}$ be a subspace of $Y$ and $(x, y) \in X \times Y$. Then, $\left(w_{0}, g_{0}\right) \in$ $P_{W_{1} \times W_{2}}^{2}(x, y)$ if and only if $\left(x-w_{0}, y-g_{0}\right) \perp W_{1} \times W_{2}$.

Proof. Note that, $\left\|x-w_{0}+\lambda_{1} w, y-g_{0}+\lambda_{2} g\right\| \geq\left\|x-w_{0}, y-g_{0}\right\|$, for all $(w, g) \in W_{1} \times W_{2}$ and all scalars $\lambda_{1} \lambda_{2}$ if and only if $\left(w_{0}, g_{0}\right) \in P_{W_{1} \times W_{2}}^{2}(x, y)$.

\section{REFERENCES}

[1] S. Gähler, Lineare 2-normierte raume, Math. Nachr., 28 (1964), 1-43.

[2] M. Açikgöz, A review on 2-structures, International Journal of Math. Analysis, Vol. 1, No. 4 (2007), 187-191.

[3] M. Açikgöz, ع-approximation in Generalized 2-normed Spaces, Matematički Vesnik, Vol. 61, No. 2, (2009), 159-163.

[4] A. White, 2-Banach spaces, Math. Nachr., 42 (1969), 43-60.

[5] Y.J. Cho, R.W. Freese, Isometry conditions in linear 2-normed spaces, Math. Japonica, Vol. 35, No. 5 (1990), 985-990.

[6] R. Freese, Y. Cho, Geometry of Linear 2-normed Spaces, Nova Science Publishers, 2001.

[7] Z. Lewaandowska, Linear operators on generalized 2-normed spaces, Bull. Math. Soc. Sci. Math. Roumanie, (N.S.) 42 (90), No. 4 (1999), 353-368.

[8] Z. Lewaandowska, On 2-sets, Glas. Math. Ser. III, No. 1 (2003), 99-110.

[9] Z. Lewaandowska, Generalized 2-normed spaces, Stuspskie Prace MatematycznoFizyczne, 1 (2001), 33-40. 
[10] Lewaandowska, Z.,(2004), Bounded 2-linear operators on 2-normed sets, Glas. Mat. Ser. III, 39 (59), 301-312.

[11] Z. Lewaandowska, Banach Steinhaus theorems for bounded linear operators with values in a generalized 2-normed space, Glas. Mat. Ser. III, 38 (58), No. 2 (2003), 329-340.

[12] S. Cobzas, C. Mustuta, Extension of bilinear functionals and best approximation, Studia Univ. Babes-Bolyai Math., Vol. 43, No. 2 (1998), 1-13.

[13] S. Elumalai, A. Asha, Patricia Jennifer, Some results on linear 2-normed spaces, Bull. Cal. Math. Soc., Vol. 92, No. 6 (2000), 441-454.

[14] S. Elumalai, M. Souruparani, A characterization of best approximation and the operators in linear 2-normed spaces, Bull. Cal. Math. Soc., Vol. 92, No. 4 (2000), 441-454.

[15] S.N. Lal, S. Bhattacharya, C. Sreedhar, Complex 2-normed linear spaces and extension of linear 2-functionals, Z. Anal. Anwendungen, Vol. 20, No. 1 (2001), 35-53.

[16] Sh. Rezapour, Proximinal Subspaces of 2-normed Spaces, Anal. Theory Appl., Vol. 22, No. 2 (2006), 114-119.

[17] Sh. Rezapour, 2-proximinality in Generalized 2-normed spaces, Southeast Asian Bull. Math., Vol. 33 (2009), 109-113.

[18] Sh. Rezapour, Best Approximation in Cone Metric Spaces, Mathematica Moravica, Vol. 11 (2007), 85-88.

Mehmet AÇIKgöz

Department of Mathematics

Faculty of Science and Arts

University of GazianteP

27310 GaZianteP

TURKEY

E-mail address: acikgoz@gantep.edu.tr 